\title{
Searching for the in-plane Galactic bar and ring in DENIS
}

\author{
M. López-Corredoira ${ }^{1}$, P. L. Hammersley ${ }^{1}$, F. Garzón ${ }^{1,2}$, A. Cabrera-Lavers ${ }^{1}$, N. Castro-Rodríguez ${ }^{1}$, \\ M. Schultheis ${ }^{3}$, and T. J. Mahoney ${ }^{1}$ \\ 1 Instituto de Astrofísica de Canarias, 38200 La Laguna, Tenerife, Spain \\ 2 Departamento de Astrofísica, Universidad de La Laguna, Tenerife, Spain \\ 3 Institut d'Astrophysique de Paris, 75014 Paris, France
}

Received 21 February 2001 / Accepted 10 April 2001

\begin{abstract}
New evidence for a long thin Galactic bar (in contradistinction to the bulge), as well as for the existence of the ring and the truncation of the inner disc, are sought in the DENIS survey. First, we examine DENIS and Two Micron Galactic Survey star counts for the characteristic signatures of an in-plane bar and ring. The star counts in the plane for $30^{\circ}>l>-30^{\circ}$ are shown to be highly asymmetric with considerably more sources at positive than at negative longitudes. At $|b| \approx 1.5^{\circ}$, however, the counts are nearly symmetric. Therefore, the asymmetry is not due to the disc, which is shown to have an inner truncation, or to the bulge, so there has to be another major component in the inner Galaxy that is causing the asymmetries. This component provides up to $50 \%$ of the detected sources in the plane between the bulge and $l=27^{\circ}$ or $l=-14^{\circ}$. This component is shown to be consistent with an in-plane bar with a position angle of $40^{\circ}$ and half-length of $3.9 \mathrm{kpc}$. However, there is also a major peak in the counts at $l=-22^{\circ}$, which coincides with the tangential point of the so-called $3 \mathrm{kpc}$ arm. This is shown to be most probably a ring or a pseudo-ring. The extinction in the plane is also shown to be asymmetric with more extinction at negative than at positive longitudes. For $l<8^{\circ}$ the extinction is shown to be slightly tilted with respect to $b=0^{\circ}$ in the same manner as the HI disc. We conclude that the Galaxy is a fairly typical ringed barred spiral galaxy.
\end{abstract}

Key words. Galaxy: general - Galaxy: stellar content - Galaxy: structure - infrared: stars

\section{Introduction}

De Vaucouleurs $(1964,1970)$ first suggested, in an attempt to explain observed non-circular gas orbits, that the Galaxy might be barred. Since then, a large body of observational evidence has been accumulated that supports this hypothesis (see Garzón 1999 for a review). Axial asymmetries in the inner Galaxy have been detected in star counts (Nakada et al. 1991; Weinberg 1992; Whitelock et al. 1991; Stanek et al. 1994; Hammersley et al. 1994, 1999, 2000; Wózniak \& Stanek 1996; Nikolaev \& Weinberg 1997; Unavane \& Gilmore 1998; López-Corredoira et al. 1997, 2000) and by surface photometry at different wavelengths (Blitz \& Spergel 1991a; Weiland et al. 1994; Dwek et al. 1995; Freudenreich 1998), microlensing (Stanek 1995; Binney et al. 2000) and analysis of internal motions of the gas (Peters 1975; Liszt \& Burton 1980; Yuan 1984; Nakai 1992; Gerhard 1996). Various models have been constructed to explain the observed features, many of which include a bar (e.g., Binney et al. 1991; Weiner \& Sellwood 1999). The observed non-axisymmetry, however, varies considerably between the various papers, although

Send offprint requests to: M. López-Corredoira, e-mail: martinlc@ll.iac.es "bar" is the term normally used. Unfortunately, this terminology is ambiguous and has led to much confusion and controversy, as has been commented on in several papers (Kuijken 1996; Ng 1998; López-Corredoira et al. 1999). This is more than a mere question of words, however. Many authors have classed what is a short fat structure as a bar, when possibly a more appropriate term would be a triaxial bulge. Other authors have found a long thin structure, which, if it exists, would be what is traditionally considered to be a bar, and such a feature is inherently different from a triaxial bulge.

Whilst evidence for the triaxial bulge is now overwhelming and the results are basically consistent, there is still discussion on the presence of the long thin bar. Radio maps show significant non-axisymmetry in the motions of the gas in the inner galaxy. Peters (1975) analysed the HI maps and showed that a bar inclined at about $45^{\circ}$ to the Sun-Galactic Centre line would produce the features seen. Nakai (1992) found a similar angle when analysing CO maps, and a rough calculation places the ends of the bar at $l \sim 30^{\circ}$ and $l=-20^{\circ}$.

$I R A S$ star counts in the plane have a very asymmetric distribution in longitude (e.g. Garzón 1999), with more stars at positive than at negative longitudes. These 
asymmetries stretch from about $l=30^{\circ}$ to $-30^{\circ}$ and are far larger than the asymmetries caused by a triaxial bulge, in both extent and magnitude. Weinberg (1992) analysed the IRAS star counts in the plane and proposed that there was an in-plane stellar bar with a position angle of $36 \pm 10^{\circ}$ and half-length of about $5 \mathrm{kpc}$. Hammersley et al. (1994, hereafter H94) examined data from the Two Micron Galactic Survey (TMGS) and showed that there is a high density of young stars in the plane at $l=27^{\circ}$ and $21^{\circ}$ that is not seen either towards the bulge or at longitudes greater than $l=27^{\circ}$. An analysis of the $C O B E / \mathrm{DIRBE}$ 2.2- $\mu \mathrm{m}$ surface brightness maps showed that the form was consistent with an in-plane bar.

Further evidence for the bar at positive longitudes is presented by Garzón et al. (1997) and López-Corredoira et al. (1999), who made a spectroscopic analysis of the brightest stars in an infrared-selected sample of objects close to the Galactic plane at $l=27^{\circ}$ showing a strikingly high fraction of supergiants, characteristic of a strong star formation region. The typical distance to these sources was found to be $6 \mathrm{kpc}$. It was argued that this result is consistent with this region being the near end of the Galactic bar. Such regions can form due to the concentrations of shocked gas where the stellar bar meets the disc, as is observed at the ends of the bars of face-on external galaxies (Sandage \& Bedke 1994). Hammersley et al. (2000, hereafter H00) examined infrared colourmagnitude diagrams in a number of regions in the plane. They show that there is a major cluster of $\mathrm{K}-\mathrm{M}$ giants at a distance of $5.7 \pm 0.5 \mathrm{kpc}$ at $l=27^{\circ}$, which is not present at $l=32^{\circ}$. This cluster is seen at most longitudes smaller than $l=27^{\circ}$ (the exception being regions of extremely high extinction) and the distance from the Sun to the cluster increases with decreasing longitude until about $l=10^{\circ}$, where it merges with the bulge. H00 argue that what is being seen at $l=27^{\circ}$ is the older component at the near end of a bar with a position angle of $43 \pm 7^{\circ}$ and half length about $4 \mathrm{kpc}$. This would put the far end of the bar near $l=-12^{\circ}$, and the authors note that there is a peak in the $C O B E / \mathrm{DIRBE}$ surface brightness maps at this location.

Other authors, however, prefer to explain these extra stars seen in the plane as belonging to a ring or spiral arms. Kent et al. (1991) suggested a model containing a thick ring and disc which did successfully reproduce the surface brightness maps at positive longitudes (their data set was principally for positive longitudes). However, their model is basically symmetrical and so would not reproduce the negative longitudes as seen in $C O B E / D I R B E$. Even making the ring elliptical would not significantly improve the fit. In fact none of the proposed models containing a bulge (including its triaxiality) disc and ring successfully reproduces the star counts or surface brightness maps in the plane between $l=40^{\circ}$ and $-40^{\circ}$. Freudenreich (1998) has significant residuals in the plane between $l=30^{\circ}$ and $-30^{\circ}$ after subtracting the disc and bulge. He prefers to attribute these residuals to patchy star formation trailing from a ring or spiral arm, however the discovery of older stars in H00 rules out patchy star formation; furthermore, the residuals are not "patchy" but almost constant between $l=30^{\circ}$ and $-12^{\circ}$.

Weiner \& Sellwood (1999) produced a gravitational model that included a separate bar and bulge, and with this model they fitted the observed features caused by the gas kinematics in the inner regions of Milky Way. Their bulge is axisymmetric and the inclination of the bar is $\sim 35^{\circ}$. In that paper, the authors claim that their treatment of bulge and bar separately does not imply that both components are different, either photometrically or kinematically. However, although they do not make such a claim, it is evident that the model works much better by introducing these two types of density distributions. Even if they were part of the same structure, the central kiloparsec would be significantly different from the outer regions.

Generally, the observations indicate that size and form of the asymmetry in the star distributions vary with Galactic latitude. Normally, when the low latitudes are excluded (i.e. observing the triaxial bulge) an angle of about $25^{\circ}$ is found. When the plane regions are included, however, the angle reaches $45^{\circ}$ (Sevenster et al. 1999). The difference in counts between positive and negative longitudes is also larger close to the plane but it can still be detected ten or more degrees from the plane. This arrangement is consistent with a triaxial bulge with an inclination around $20^{\circ}$ or less, dominating the off-plane regions, and a thin bar with an inclination around $45^{\circ}$, which gives strong asymmetry only in in-plane positions. The change in angle with latitude would be related to the ratio of bulge and bar counts, with each having different position angles.

An important parameter in any barred galaxy is the location of corotation. Corotation is at 1.1 to 1.2 times the length of the bar and the bar cannot extend beyond corotation. However, a triaxial or boxy-peanut bulge will end near the vertical ILR, which is at about $R_{\mathrm{cr}} / 2.2$ (Friedli 1999). Therefore, both the thin bar and triaxial bulge put corotation at about 4.4 to $5 \mathrm{kpc}$. This is in agreement with the conclusions of Combes (1996). Galaxies with two, or even three, triaxial structures are known (see Friedli 1996 for a review) and the position angles of the various components can take any value (in fact, they are aligned only in a minority of cases). Hence, it is quite reasonable that a triaxial bulge and in-plane bar have slightly different position angles.

From the above it is clear that the non-axisymmetry in the inner Galaxy is best described by two separate components: a "triaxial bulge" and a "thin bar" and the two should not be confused. The bar is long and thin, and contains both young and older stars, whereas the triaxial bulge is shorter, far fatter and only contains old stars (H94, H00, Garzón et al. 1997; López-Corredoira et al. 2000). This implies that the inner Galaxy when viewed face on would appear similar to M 95 (NGC 3351), of revised Hubble type SBb(r)II (Sandage \& Tammann 1981) (Fig. 1), although the bulge of the Milky Way may be less 


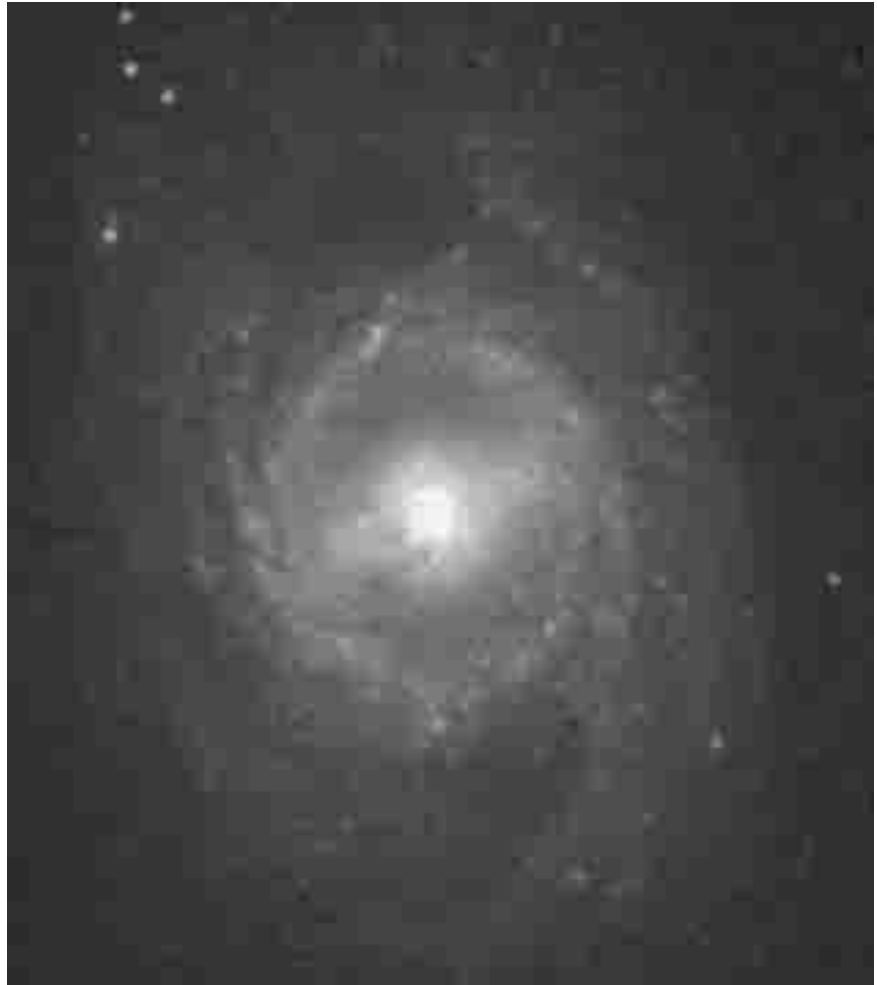

Fig. 1. POSSII(J-Blue) image of the spiral galaxy M 95 (NGC 3351). Note the presence of a bar apart from the prominent bulge in the centre of the galaxy.

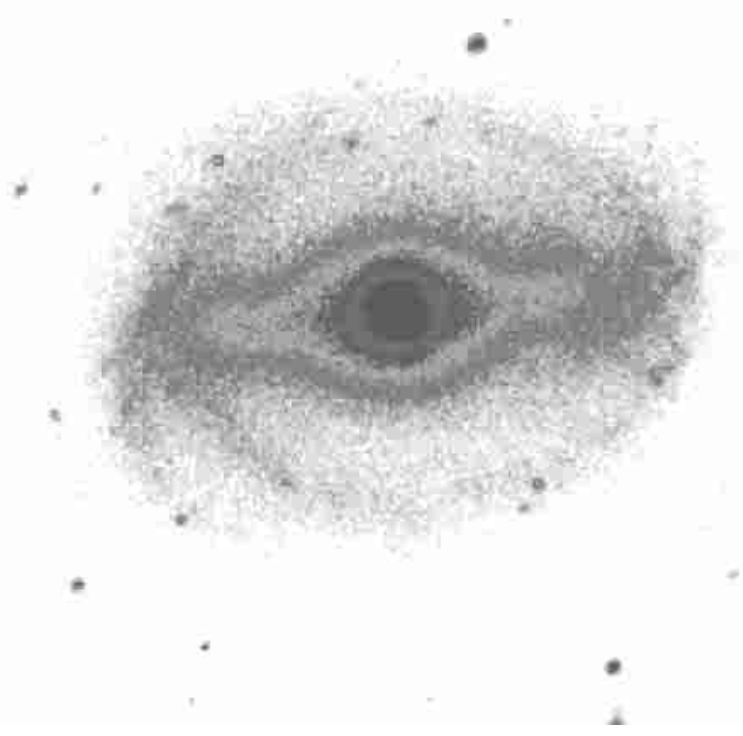

Fig. 2. POSSII(R-Red) image of the spiral galaxy NGC 1433. Another example of a barred galaxy with bulge.

prominent, or NGC 1433 (Fig. 2), revised Hubble type SBb(s)I-II (Sandage \& Tammann 1981), where "r" denotes "ringed" and "s" denotes that the spiral arms originate either from the ends of the bar or the from the centre, and the roman numerals I and II denote early

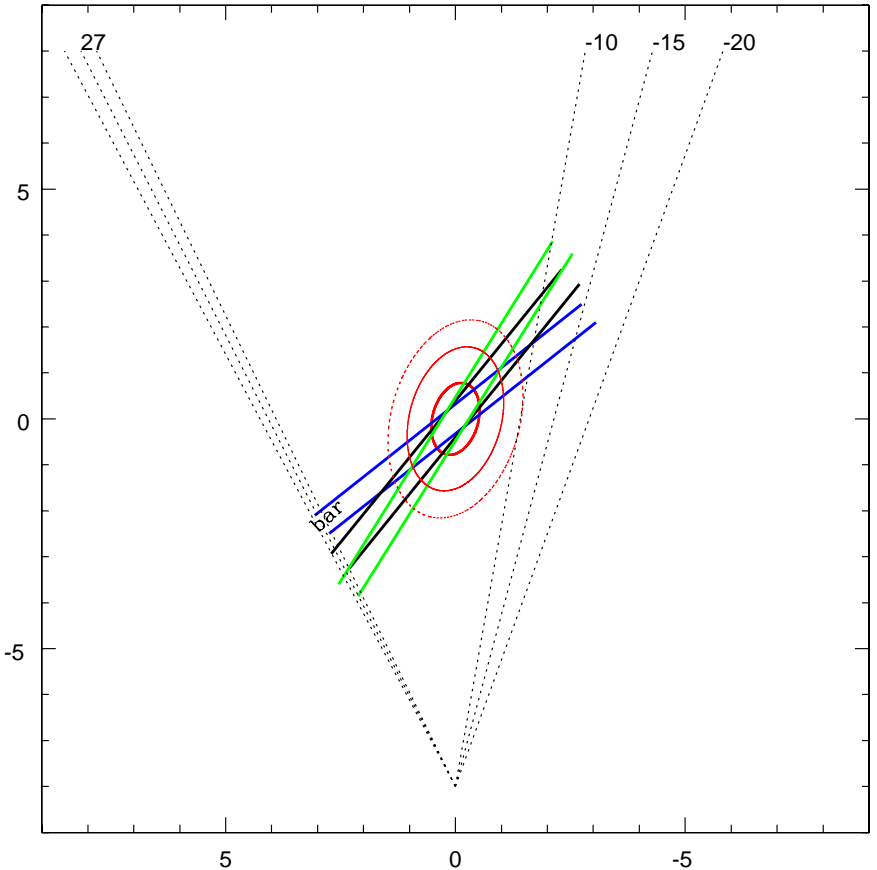

Fig. 3. A Schematic representation of the proposed "triaxial bulge" + "bar" structures in the inner Galaxy. The bar width is assumed to be $500 \mathrm{pc}$. Three possible bars are plotted showing the range of position angles determined from $\mathrm{H} 00$ and assuming that the near end of the bar is at $l=27^{\circ}$. The lines of sight for $l=26^{\circ}, 27^{\circ}, 28^{\circ},-10^{\circ},-15^{\circ},-20^{\circ}$ are drawn. The Sun is at $(0,-8) \mathrm{kpc}$.

type and intermediate luminosities. Other authors interpret NGC 1433 in terms of a double-barred galaxy (Friedli 1999) with different assumptions (they assume a elliptical shape for the thin bar instead of a sticklike shape); in any case, we have two different non-axisymmetric structures. Figure 3 shows a graphical representation of the proposed configuration for the inner Galaxy. It includes:

A long, thin bar (half-length $3.9 \mathrm{kpc}$ ) with the near end at $l=27^{\circ}$. Three possible values for the angle between the bar and Sun-Galactic Centre line are shown in Fig. 3: $50^{\circ}, 43^{\circ}$ and $36^{\circ}$. Such a bar would have star formation regions at its tips;

A triaxial bulge, which is shorter, far thicker (extending to $|b| \approx 10-15^{\circ}$, with a typical angle of about $12^{\circ}$ with respect to the Sun-Galactic centre line, and containing only old population stars (except for a small region in the inner bulge, López-Corredoira et al. 2001a).

The aim of this paper is to examine DENIS star counts for evidence of the long, thin bar and/or ring. Both the proposed continuous ring and bar make clear predictions as to the form of the star counts in the inner Galaxy, which can be tested against the data. In this paper, the term "bar" will refer only to the in-plane bar described in H00. When discussing the non-axisymmetry of the bulge, the term "triaxial bulge" will be used. The evidence for asymmetry in in-plane regions is explored in this paper 
through the analysis of star counts (Sect. 3) and extinction (Sect. 4).

\section{DENIS data}

The Deep Near-Infrared Survey of the southern sky (DENIS) is a survey the southern hemisphere in three bands: Gunn $I(0.82 \mu \mathrm{m}), J(1.25 \mu \mathrm{m}), K_{\mathrm{s}}(2.15 \mu \mathrm{m})$ with limiting magnitudes $18.5,16.5$ and 14.0 and saturation magnitudes 9.5, 8.5 and 6.5, respectively (Epchtein 1998). The ESO $1 \mathrm{~m}$ Telescope at La Silla (Chile) was used for the survey. The observations were begun in 1995 December and will be completed by the end of 2001 approximately. Part of these data are already reduced and a small part has already been released at the CDS for public use (Epchtein et al. 1999). A special effort has been made to cover priority regions of high scientific interest, such as the inner Galactic plane and bulge, and it is these data that will be used in this paper. In total, an area of around $170 \mathrm{deg}^{2}$ within the area $|l|<35^{\circ},|b|<2^{\circ}$ has been covered. As the positive longitudes were less sampled than the negative longitudes $\left(96 \mathrm{deg}^{2}\right.$ are available at $-35^{\circ}<l<0^{\circ},|b|<1.5^{\circ}$, while for positive longitudes only half of the area is covered: $47 \mathrm{deg}^{2}$ are available at $0^{\circ}<l<35^{\circ},|b|<1.5^{\circ}$ ) some data from TMGS (Garzón et al. 1993, 1996) were used to complete the counts in those regions without DENIS data, and to calculate the star counts of the Sect. 3. With regard to the extinction shown in Sect. 4, the calculations were carried out from DENIS data by Schultheis et al. (private communication).

The images were reduced at the Paris Data Analysis Centre (PDAC) of DENIS, and homogeneous criteria of reduction were applied to the whole special sample of the bulge, which is used here. They were checked and calibrated (Borsenberger 1997; Ruphy et al. 1997) at PDAC. The point source extraction was also carried out at PDAC by fitting PSFs optimized for crowded fields. For the astrometry, the individual DENIS frames were crosscorrelated with the PMM catalogue (USNO-A2.0) with a precision of better than one arcsecond.

\section{Asymmetry in the star counts of the Galactic plane}

Star counts provide a powerful tool when searching for asymmetries in the stellar distribution in the inner Galaxy. Other researchers have already used this method (see introduction); however, they have mostly observed regions where the bulge is predominant. While the bulge is observed in off-plane regions up to $b \sim 10$ degrees and in $|l|<15$ degrees, the long, thin bar is visible only in the in-plane regions, $|b|<2$ degrees and up to $|l|=27^{\circ}$ at positive longitudes, and somewhat less at negative longitudes. It is now well known that the star counts in the bulge are non-axisymmetric (e.g. López-Corredoira et al. 2000); however, the larger-scale in-plane asymmetries are less well investigated.
The most appropriate DENIS filter for probing the inner Galaxy near plane is $K_{\mathrm{s}}$, since the effect of extinction is much lower than in the other filters. However, the other filters can be used to provide information about the population of the stars and the extinction.

Although the nominal $K_{\mathrm{s}}$ limiting magnitude of DENIS is around 14.0 (Epchtein 1998); the presence of confusion implies that in the more crowded regions the completeness can be two or three magnitudes brighter, particularly towards the inner bulge (Unavane et al. 1998). For this study, however, only the brighter stars will be used, so confusion is not an issue. Whilst it is often argued that the fainter the star counts the better, this is not necessarily so when observing the inner Galaxy. The ratio of inner Galaxy to local disc stars is more important. At magnitude 13 or 14 there will be more inner Galaxy sources than at magnitudes 8 to 10 , but the numbers of local disc sources rises even more rapidly. Garzón et al. (1993) showed that the best contrast when looking at the bulge was at near $m_{K}=+8$, and that at fainter magnitudes the contrast became poorer. However, the number of stars detected falls rapidly much brighter than this (few if any bulge sources are brighter than $m_{K}=6$, L00) and the statistical errors become important. For this paper star counts up to $m_{K_{\mathrm{s}}}=9$ will be used, as the inner-Galaxyto-disc contrast is high, but there are still sufficient sources to provide good statistics.

The star counts down to $m_{K_{\mathrm{s}}}=9.0$ are shown in Fig. 4 . The DENIS star counts show the average of the available counts in a region of width $\Delta l=5^{\circ}$ centred on the position marked. A large bin size was adopted, as this averages out the fluctuation due to the varying extinction and the asymmetries being sorted are distributed over many tens of degrees. To complete the longitude coverage, the star counts from TMGS are used (see Hammersley et al. 1999 for more details on the data). The TMGS data, however, are plotted at the average longitude where the strip crossed the plane. In both cases the areas of sky covered were large so that the Poissonian error is negligible. There is a difference between the standard $K$ filter of the TMGS and the DENIS $K_{\mathrm{s}}$; however, Cohen (1997) has shown that the difference between the two for any star is less than $0.03 \mathrm{mag}$, so the effect on the counts of using one or other should be negligible.

Figure 4 shows five slices across the plane at $2^{\circ}>b>$ $1.5^{\circ}, 1^{\circ}>b>0.5^{\circ}, 0.25^{\circ}>b>-0.25^{\circ},-0.5^{\circ}>b>-1^{\circ}$ and $-1.5^{\circ}>b>-2^{\circ}$. Therefore, at the distance of the Galactic Centre $(8 \mathrm{kpc})$ the average heights over the plane are $0 \mathrm{pc}, \pm 100 \mathrm{pc}$ and $\pm 240 \mathrm{pc}$. It is noticeable that the cuts furthest from the plane are substantially different from those in the plane. It can further be seen that the counts are more or less symmetrical in latitude. The slight differences that there are can be attributed to the Sun being about $14 \mathrm{pc}$ above the Galactic plane (Hammersley et al. 1995), which would give more counts at negative than at positive latitudes, and differences in the extinction above and below the plane. The small differences that there are between the TMGS and DENIS counts can be 


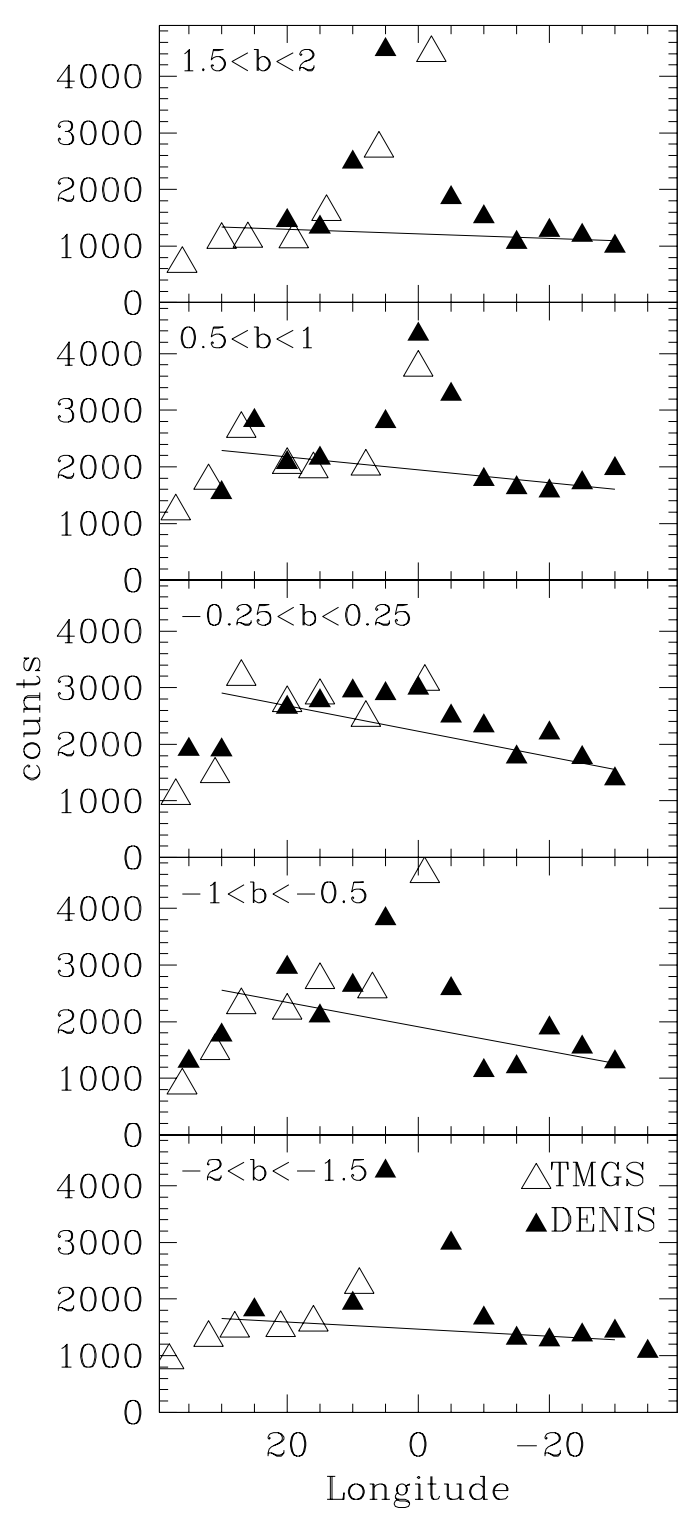

Fig. 4. Star counts with $m_{K} \leq 9.0$ at different Galactic latitudes. The crosses are data from the DENIS survey (Epchtein 1998) averaged over $\Delta l=5^{\circ}$, and the circles are from TMGS (Garzón et al. 1993, 1996). Large regions were used for each region with thousands of stars, so the Poissonian error is negligible. Dashed lines show a general trend in the star counts in $10^{\circ}<|l|<25^{\circ}$.

attributed to the fact that they are centred on different longitudes and cover different areas of sky.

\subsection{Off-plane star counts}

Before attempting to disentangle the $b=0^{\circ}$ slice, it is important to understand the shape of the disc, as this will be an important feature in the plane. The slices at $|b|=1.75^{\circ}$ should be very sensitive to the shape of the disc. They are far enough from the plane that the extinction will be low, typically 2 to 3 magnitudes in the visible or 0.2 to $0.3 \mathrm{mag}$ at $K_{\mathrm{s}}$. Furthermore, the lines of sight run sufficiently far above and below the plane that the young components

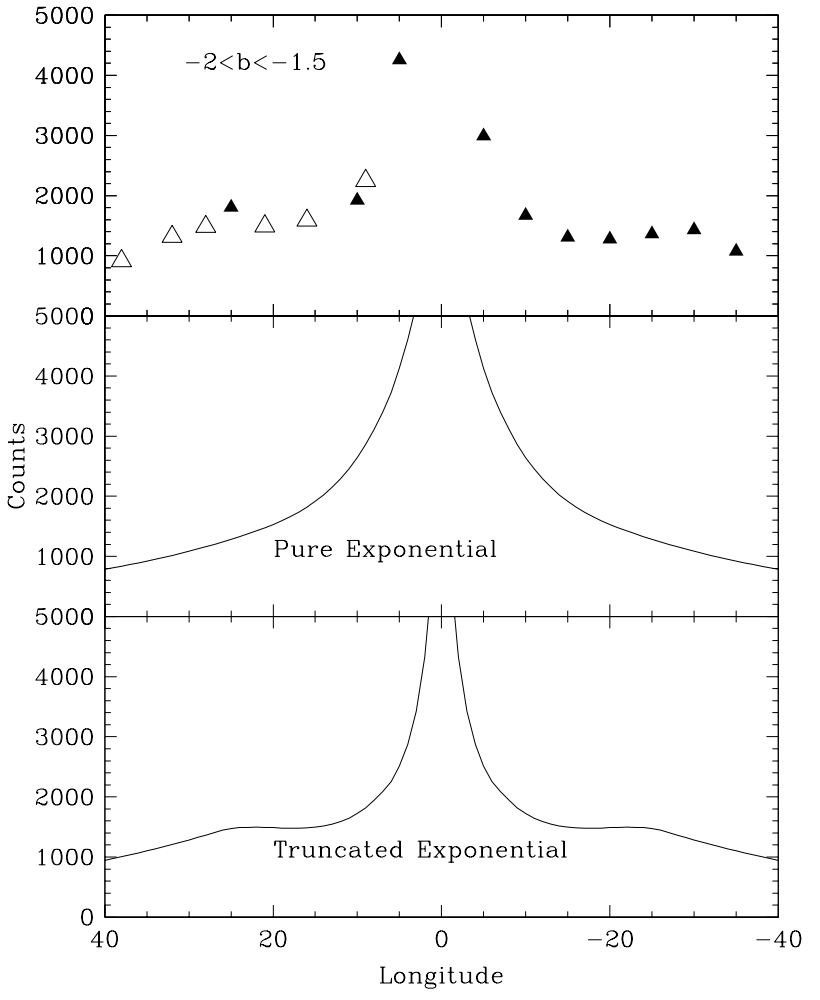

Fig. 5. Star counts with $m_{K} \leq 9.0$ for $-1.5^{\circ}>b>-2^{\circ}$ and the predicted counts using two simple disc-bulge models (see text).

close to the plane will not be a major component in the counts and only inner Galaxy features with scale heights in the hundreds of parsecs should provide a significant contribution.

Figure 4 shows that the slices for $|b|=1.75^{\circ}$ are more or less symmetric in longitude. Initially, the counts rise with decreasing longitude but then for $30^{\circ}>|l|>12^{\circ}$ the counts are basically flat before rising steeply for $|l|<10^{\circ}$ as the bulge counts become increasingly important. These plateaux are not expected for a purely exponential disc. Figure 5 shows the counts for $-1.5^{\circ}>b>-2^{\circ}$ and the predicted counts using two simple disc-bulge models. Both models are based on Wainscoat et al. (1992), whose model has been shown to reproduce the TMGS star counts very well (Hammersley et al. 1999). The bulge used is that described in Wainscoat et al. (1992); the fact that the model is axisymmetric whereas the bulge is triaxial is not important for the following discussion. The extinction model used is also as described in Wainscoat et al. (1992). The difference between the two plots is that the figure marked "exponential disc" has an exponential disc that continues into the Galactic Centre, again as in Wainscoat et al. (1992). However, the model marked "truncated exponential" has a stellar density that only rises exponentially inwards down to $3.5 \mathrm{kpc}$, from which point it falls off linearly such that at the centre it is zero. This approximates to a Freeman type II disc with a central hole and is known as an inner truncated disc. 
The model predicts that a purely exponential disc that continues into the centre should rise increasingly steeply with decreasing longitude; however, the measured counts clearly do not do this. The truncated model does, however, reproduce the shape very well. A full discussion of the exact form of the inner disc is beyond the scope of this paper, as data further from the plane are required; however, this result is in agreement with the findings from the analysis of the $C O B E / D I R B E$ surface brightness maps (e.g. Freudenreich 1998). Inner truncated discs are very common in barred spirals. Ohta et al. (1990) looked at six early-type spirals and found that all had Freeman type II discs when looking perpendicular to the plane. Baggett et al. (1996) show that barred galaxies are a factor of two, or more, more likely than non-barred Galaxies to have an inner truncated disc. They also note that an increasing number of bars is being found in galaxies previously classified as non-barred, so this percentage is likely to rise.

\subsection{In-plane star counts}

The large-scale asymmetry counts in the plane are very noticeable. Positive longitudes consistently have far more counts than negative longitudes. Furthermore, the shape of the counts is very different at positive and negative longitudes, so the form cannot be explained by simply making the inner Galaxy elliptical. From about $l=27^{\circ}$ to the Galactic Centre the counts are flat, whereas between $l=0^{\circ}$ and $l=-18^{\circ}$ they are reduced by a factor 2 . This asymmetry is greater in the $b=0^{\circ}$ strip than in the $|b|=0.75^{\circ}$ strip because the relative contribution of the bar will be larger at $b=0^{\circ}$. The disc will contribute about $50 \%$ of the counts at $l=20^{\circ}$ in the plane but, as is shown in the previous section, the disc is symmetric. Hence, when the disc is subtracted from the in plane counts the asymmetries between positive and negative longitudes in the remaining counts (i.e. those from the inner Galaxy components) become enormous.

The peak due to the bulge at $l=0^{\circ}$ is hardly seen at all in the in-plane strip, whereas it is clearly evident in the off-plane counts. This is due in part to the strong extinction within a few hundred pc of the Galactic Centre (GC) which drastically reduces the counts in the plane (Hammersley et al. 1999). Hence, the rapid increase in star density near the GC is masked. However, the $l=7^{\circ}$ $b=0^{\circ}$ line of sight runs far enough away from the GC not to be affected by the very high extinction near the GC, and here the bulge contributes about $50 \%$ of the counts to $m_{K}=9$ (Fig. B2 in Hammersley et al. 1999). At greater absolute longitudes the bulge quickly dies away, providing a negligible contribution for $|l|>10^{\circ}$. Therefore, there has to be another component in the plane taking its place at greater absolute longitudes to make the counts almost flat up to $l=27^{\circ}$, where it then stops quite suddenly. This component contributes around $50 \%$ of the detected sources, so it must be a major feature in the inner Galaxy. This component is principally seen at positive longitudes,

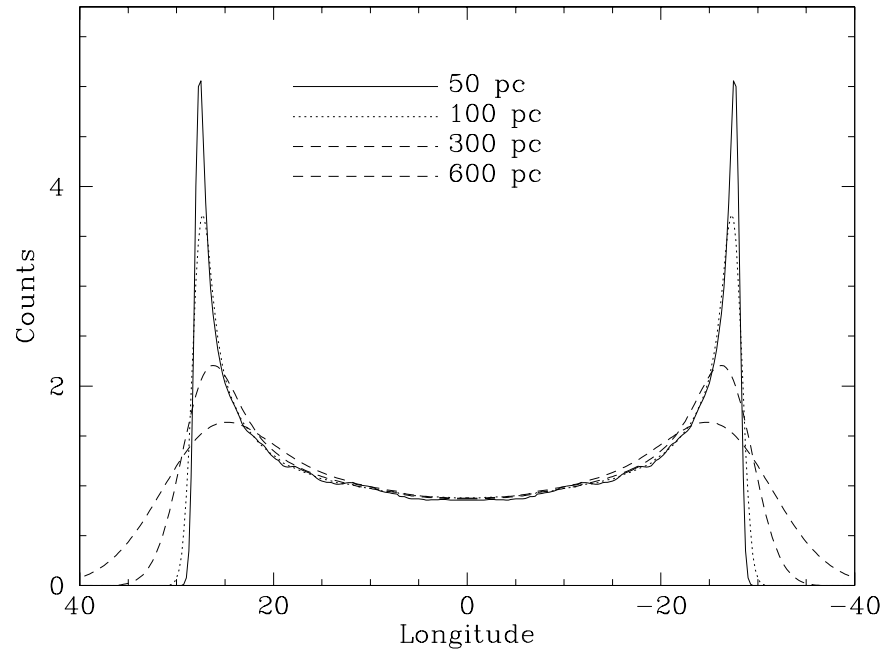

Fig. 6. The expected shape of the counts produced by rings of varying thicknesses for a cut along the plane. The radius of the ring is $3.7 \mathrm{kpc}$ and the radial distribution is Gaussian with the stated sigmas. The counts were normalized such that each ring would give the same counts at $l=0^{\circ}$. The ring used was circular; if it were elliptical then the effect would be to change the position of the peaks so that they were no longer symmetric in longitude.

extending from the bulge up to $l=27^{\circ}$ and somewhat less at negative longitudes. This makes the component extremely asymmetric as seen from the Sun, far more so than the triaxial bulge.

Clearly, one explanation for the asymmetry in the counts would be extinction. This has an important role as a bar would be expected to have dust lanes on its leading edges (Calbet et al. 1996) and will be discussed in Sect. 4; however, extinction can only reduce the numbers of stars, whereas here there is clearly an extra population in the plane.

In H94, a ring, by itself, is shown not to explain the in-plane features. A ring would be expected to produce a peak in the counts at the positions tangential to the line of sight. If the ring is relatively thin, then away from the tangential points the counts should die away quickly. If the ring is very broad (e.g. as proposed by Kent et al. 1991), then the peak becomes a lot broader. Figure 6 shows some possible forms that the ring counts could produce for an axially symmetric system. If the ring were elliptical then the longitudes of the peaks would no longer be symmetric; however, the shapes of the peaks would remain basically unaltered. Clearly, it is possible that a contrived ring (particularly a patchy ring), coupled with a highly improbable distribution of extinction, could reproduce the form of the in-plane counts. However, as will be shown, this distribution of extinction does not exist and hence the ring by itself cannot explain the in-plane counts.

The other alternative to explain the asymmetry in the star counts is the existence of a bar. The work in H94 and H00 limits the possible orientations of the bar. The predicted bar has the near end at $l=27^{\circ}$ at a distance of $5.7 \mathrm{kpc}$ and the far end at $l=-12^{\circ}$ at a distance of 
about $11 \mathrm{kpc}$. We shall limit the discussion to whether the data presented here are consistent with the previous results rather than try to re-determine all the parameters, as the arguments presented would be almost identical.

Whilst simplistically one would expect the near end of the bar to give more counts than the far end, this is not necessarily the case in the plane. Blitz \& Spergel (1991b) showed that the far end of the bar can give a higher surface brightness than the near end in the plane; Unavane et al. (1998) and Unavane \& Gilmore (1998) predict a similar result for star counts. There are, however, a series of effects that have to be taken into account:

- The number of stars per unit area of sky is dependent on the volume of the bar intersected by the line of sight. This is dependent on the square of the distance to the bar at that position coupled with sec $\alpha$, where $\alpha$ is the angle between the bar and the line of sight. The regions at negative latitudes are more distant from the Sun than the regions on the positive side of the bar, and the line of sight cuts the bar at a shallower angle, both of which factors increase the counts per unit area at negative longitudes in comparison with positive longitudes;

- For the same apparent magnitude, the limiting absolute magnitude of the sources detected at positive longitudes is fainter than that at negative longitudes. Up to $m_{K}=+9$, the luminosity functions will be dominated by young stars (roughly $M_{K}<-7.5$ for a source at $l=-12^{\circ}$ and $M_{K}<-6.0$ for a source at $l=+27^{\circ}$, taking extinction into account). The luminosity function rises steeply over this magnitude range; this means that there will be far more detectable sources per unit volume at positive longitudes than at negative longitudes;

- The scale height of the bar is very important. H94 showed that the scale height for the sources in the spike at $l=21^{\circ}$ was around $50 \mathrm{pc}$. This corresponds to $b=$ $0.5^{\circ}$ for the near end of the bar at $l=27^{\circ}$ but $b=0.25^{\circ}$ for the far end. Therefore, for the $|b|<0.25^{\circ}$ counts, the edge of the box is a scale height at $l=-12^{\circ}$, only half a scale being at $l=27^{\circ}$. The effect is to reduce the "far end" / "near end" count ratio. Further from the plane the ratio is decreased even further, such that in the region $0.5^{\circ}<b<1^{\circ}$ the far end of the bar will hardly be seen, whereas at the near end it will still be visible. If the scale height were a number of degrees, as in the bulge, then the effect would be small this close to the plane;

- The far end of the bar is at twice the distance of the near end and hence the extinction is likely to be greater. Furthermore, a sticklike bar could have a dust lane on its leading edge, which would further increase the extinction at negative longitudes. This gives further counts for the positive longitudes.

Figure 7 shows the measured and model counts distribution for three latitude ranges. The model contains the truncated disc, but with the bulge and extinction based on Wainscoat et al. (1992). The simplest possible bar distribution in agreement with $\mathrm{H} 00$ was then added. The bar used has a thickness of $500 \mathrm{pc}$, a half-length of $4 \mathrm{kpc}$ and position angle of $43^{\circ}$. The distribution was assumed to be constant along the bar but exponential in height above the plane. The magnitude limit means that the sources would have to have absolute magnitudes brighter than $M_{K}=-6$. These sources will be principally young stars and so the scale height will be small. These sources will be the same as those in the spike seen in H94, which have a scale height of about $50 \mathrm{pc}$. The luminosity function used was the same as for the disc although the density was then normalized to make the total counts match those at $l=27^{\circ}$.

Whilst the model is simple, it does reproduce the measured counts fairly well, apart from the region near the GC, where the extinction model used is almost certainly not correct:

- The model predicts the sharp jump in counts near $l=27^{\circ}$ in the plane;

- The model predicts nearly constant counts in the plane between $l=27^{\circ}$ to about $l=-12^{\circ}$, at which point there is a drop to the disc level in the counts;

- In the $-1^{\circ}<b<-0.5^{\circ}$ plot, the model predicts that the extra sources are significant at positive longitudes but not at negative longitudes. The point at $l=-10^{\circ}$, $b=0.75^{\circ}$ is more or less at the level of the disc counts, whereas at $l=-10^{\circ} b=0^{\circ}$ there is a significant extra population. This is caused by the small scale height of the bar.

The only major feature not predicted is the peak at $l=$ $-22^{\circ}$. This will be dealt with later.

Unavane \& Gilmore (1998) and Unavane et al. (1998) analysed narrow-band $L$ star counts and DENIS data at a few positions in the Galactic plane, near the centre. Contrary to the results presented here, they found higher counts at negative longitude than at positive longitude, and this fits some of the models for the "bar", although, from the introduction, this could also be classed as the triaxial bulge. By observing only a few regions in the central few degrees, they were principally measuring the bulge, which dominates the star counts in the inner plane (L00). This is an old population with a scale height far greater than the $50 \mathrm{pc}$ of the feature seen here, which, as discussed above, would increase the counts at negative longitudes. In this paper, however, we are dealing with a young population extending tens of degrees along the plane from the centre. This is a very different feature, and so our results cannot be compared with those of Unavane \& Gilmore (1998) and Unavane et al. (1998).

The above shows that the DENIS + TMGS star counts to $m_{K}=+9$ between $l=27^{\circ}$ and $-12^{\circ}$ do have the characteristic signatures of the young component of a thin bar. The ring by itself, even if made elliptical, cannot give a good fit to the data (Fig. 6). Therefore, the bar proposed 


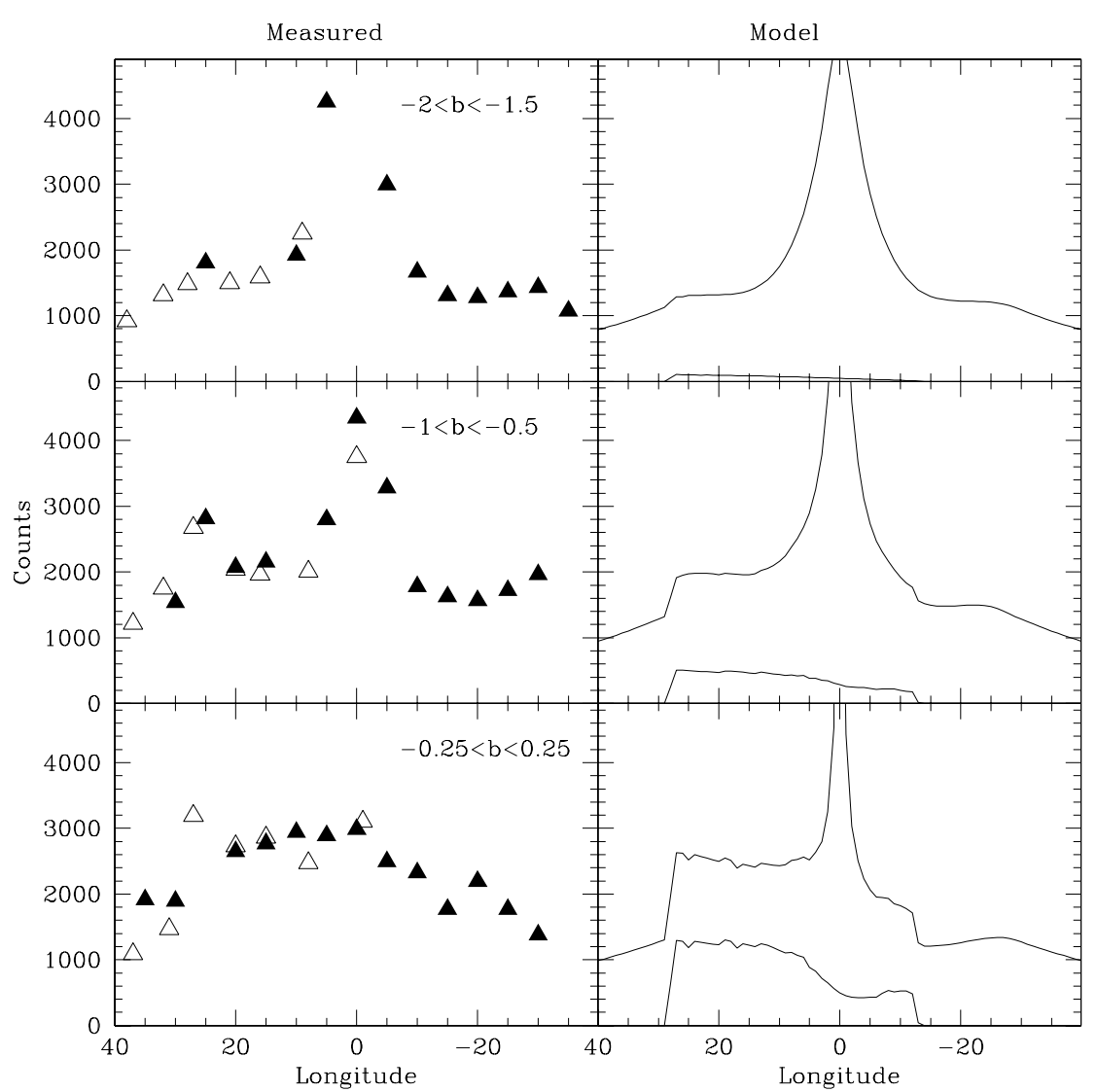

Fig. 7. The left hand plots show the measured DENIS (solid triangles) and TMGS (open triangles) star counts to $m_{K_{\mathrm{s}}}=9$ for the regions at $-2^{\circ}<b<-1.5^{\circ},-1^{\circ}<b<-0.5^{\circ}$ and $-0.25^{\circ}<b<0.25^{\circ}$. The right hand plots show the prediction of the total counts of a model containing an inner truncated disc, bulge and $3.9 \mathrm{kpc}$ bar at position angle $40^{\circ}$. The bar contribution alone is also plotted with another solid line.

in H00 correctly predicts the majority of the features seen, without resorting to ad hoc solutions.

\section{Asymmetry in the extinction of the Galactic plane}

Another way of observing possible asymmetries is to examine the distribution of extinction along the plane. The proposed bar would make the stars in the fourth quadrant significantly further away than the bar stars in the first quadrant and, consequently, the extinction of the bar stars would be higher at negative longitudes than at positive longitudes. Moreover, a possible dust lane leading the bar (Calbet et al. 1996) would further increase the extinction at negative longitudes. For the proposed bar the extra extinction would lie between where the dominance of the bulge in the counts ends $\left(l \approx-8^{\circ}\right)$ and somewhere near the far tip of the bar $\left(l \approx-12^{\circ}\right)$.

The calculation of the extinction is not straightforward and requires a number of assumptions to be made, in particular about the stellar populations. However colourmagnitude diagrams do allow a simple separation of disc dwarfs, disc giants and inner Galaxy giants when there is significant extinction along the line of sight (e.g. H00 or Ruphy et al. 1997). In DENIS, $J-K_{\mathrm{s}}$ is more appropriate since the $I$ filter does not reach the stars in the inner Galaxy along lines of sight to regions with high extinction. Using this idea, Schultheis et al. (1999) derived a method to obtain an extinction map towards the inner Galactic plane. This used $J$ and $K_{\mathrm{s}}$ DENIS bands to isolate the inner Galaxy sources together with the interstellar extinction law $\left(A_{V}: A_{J}: A_{K_{\mathrm{s}}}=1: 0.256: 0.089\right)$ from Glass (1999). Theoretical isochrones (Bertelli et al. 1994) were calculated for the RGB and AGB phase assuming a 10 Gyr population with $Z=0.02$ and distance $8 \mathrm{kpc}$. The $A_{V}$ is then determined from the shift in the colourmagnitude diagram $\left(K_{\mathrm{s}}\right.$ vs. $\left.J-K_{\mathrm{s}}\right)$ of the isochrones. This method was applied in the region $|l|<8^{\circ}$ in the paper by Schultheis et al. (1999). Here, the same method will be applied for a wider region, $|l|<20^{\circ}$. The results, after smoothing the extinction map, are shown in Figs. 8 and 9 .

Asymmetry is clearly observed in the extinction for $|b|<0.25^{\circ}$ and for $0.5^{\circ}<b<1.0^{\circ}$. In the plane there are on average two or three magnitudes more of extinction at negative longitudes than at positive longitudes. For off-plane regions $1.5^{\circ}<|b|<2.0^{\circ}$ the asymmetry in the extinction is not present. Again, a bar is consistent with this result, whereas a ring is not. The extinction calculated here is only approximate as it is impossible to isolate the stars of the inner Galaxy from those in the disc, 
Extinction

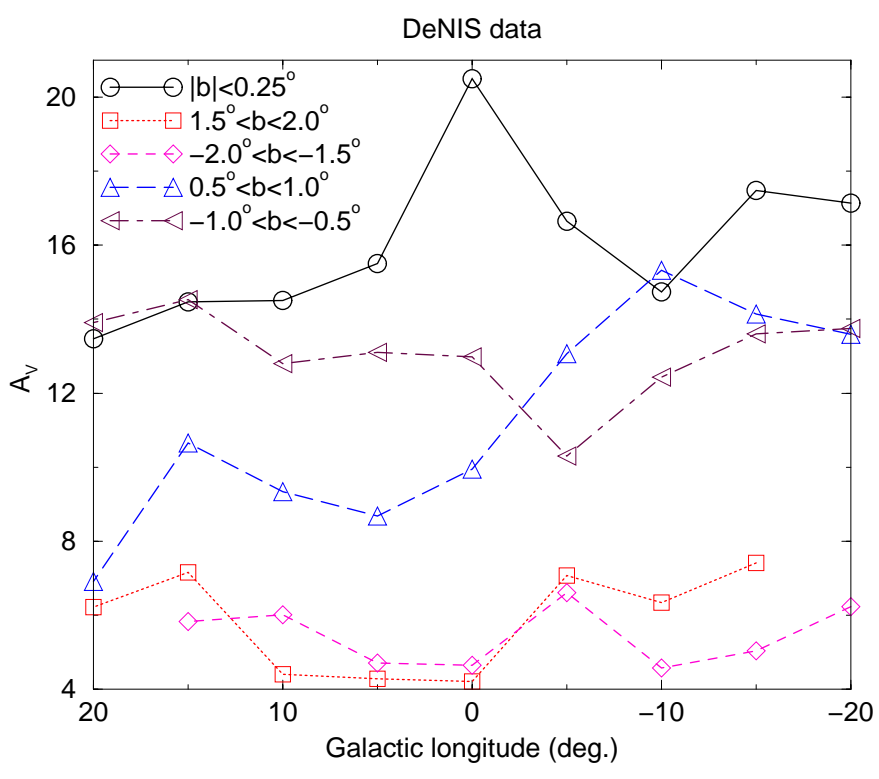

Fig. 8. Plot of the extinction $\left(A_{V}\right)$ averaged over $\Delta l=5^{\circ}$ in different latitude cuts with $\Delta b=0.5^{\circ}$ corresponding to the filter $V$ up to the furthest bulge/bar/disc stars, calculated from DENIS data by means of the method by Schultheis et al. (1999).

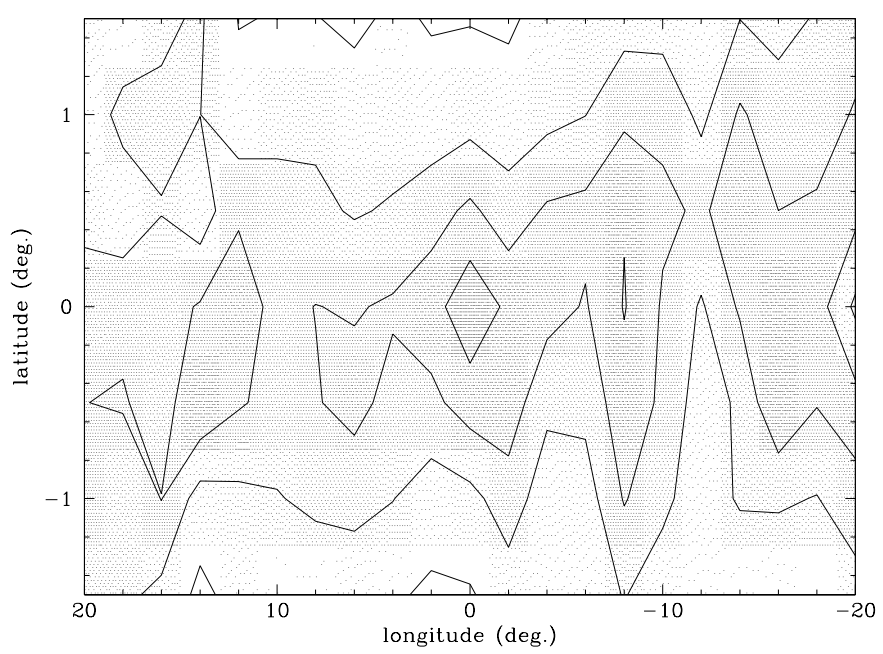

Fig. 9. Extinction map averaged and interpolated over $\Delta l=$ $2^{\circ}$ in different latitude cuts with $\Delta b=0.5^{\circ}$. Some gaps in the available data were substituted by interpolated data from the surroundings. The contours stand for the extinction $\left(A_{V}\right)$ in the filter $V$ up to the furthest bulge/bar/disc stars. This was calculated from DENIS data by means of the method of Schultheis et al. (1999). Contour step of 5 mag.

including the disc beyond the Galactic Centre. However, although the exact interpretation of the value of $A_{V}$ is not immediately obvious, the asymmetry is present and this can only be due to an intrinsic asymmetry in the central distribution of stars and/or dust.
Figure 9 presents the extinction as a two-dimensional map over the region $|l|<20^{\circ}$ and $|b|<1.5^{\circ}$. As well as the extra extinction at negative longitudes, there is a clear tilt in the extinction. This tilt follows a path line $b \approx-0.05 \times l$ for $|l|<8^{\circ}$, so it runs below the plane at positive longitudes and above the plane at negative longitudes. This is a well-known feature in the $\mathrm{CO}$ and HI maps (Liszt \& Burton 1980; Sanders et al. 1984). Observing a tilt in the extinction map clearly indicates that this extinction is due to gas and dust in the centre of the Galaxy, where the gas distribution is tilted. Because of the tilt, the asymmetry for $-1.0^{\circ}<|b|<-0.5^{\circ}$ is not seen in Fig. 8 whilst the asymmetry at $0.5^{\circ}<b<1.0^{\circ}$ is very high. At positive longitudes, the extinction is around 5 magnitudes less than at negative longitudes, except for a few isolated regions, such as the excess at $\left(l=15^{\circ}, b=-0.5^{\circ}\right)$, probably associated with a cloud in the molecular ring.

\section{An analysis of the inner Galactic plane at $l<0^{\circ}$}

A map of the star counts with $m_{K_{\mathrm{s}}} \leq 9.0$ is shown in Fig. 10 (upper) with a binning of $\Delta l=2^{\circ}$ and $\Delta b=0.5^{\circ}$. This binning reduces high frequency fluctuations due to very patchy extinction. Only the negative longitudes are shown because in this region coverage in the DENIS batches is almost complete $\left(96 \operatorname{deg}^{2}\right.$ are available at negative longitudes, while for positive longitudes only $47 \mathrm{deg}^{2}$ are available). Furthermore, previous papers have already studied the positive longitudes (e.g. H94; Kent et al. 1991), whereas there is relatively little published on the negative longitudes.

In the star-count map there are peaks in a number of regions $\left(l=0^{\circ}, b= \pm 1.5^{\circ} ; l=-5^{\circ}, b=-1^{\circ} ; l=-12^{\circ}\right.$, $\left.b=0^{\circ} ; l=-22^{\circ}, b=0^{\circ} ; l=-30^{\circ}, b=-1^{\circ}\right)$. Some of these peaks may be due to real features in the star distribution, but others are clearly due to regions of lower extinction than in the surrounding areas. Often, local maxima in the star counts are present where there are local minima in the absorption (Fig. 9). The extreme patchiness of the extinction, particularly when looking towards the inner Galaxy, means that care needs to be taken when interpreting counts in a few small areas, even when working at longer wavelengths, for example the $L$ band or $12 \mu \mathrm{m}$. The extinction can vary by 5 to 10 magnitudes in the visible over a few degrees, which will change $A_{L}$ by 0.25 to 0.5 magnitudes and hence will have a significant effect on the counts.

One method of distinguishing the peaks caused by areas of lower extinction from real features is to derive star counts corrected for extinction. The measured colour of a source is dependent on its intrinsic colour and the extinction. When looking at a restricted apparent magnitude range the majority of the sources at the same distance will have the same intrinsic colour. Hence when the majority of the sources along a particular line of sight are at the same distance, the extinction to each source - and 
hence the magnitude that the source would have without extinction - can be calculated as:

$m_{K_{\mathrm{s}}, \text { corrected }}=m_{K_{\mathrm{s}}}-\frac{A_{K_{\mathrm{s}}}}{A_{J}-A_{K_{\mathrm{s}}}}\left[\left(J-K_{\mathrm{s}}\right)-1.0\right]$

if

$\left(J-K_{\mathrm{s}}\right)>1.0$.

and

$\frac{A_{K_{\mathrm{s}}}}{A_{J}-A_{K_{\mathrm{s}}}}=\frac{3}{5}$.

The correction is calculated from the difference between the measured $\left(J-K_{\mathrm{S}}\right)$ and the expected intrinsic colour of the sources. At the distance of the bulge, $m_{K}=+9$ means that the sources are early M-giants, and so the intrinsic colour will be about 1.1 . The procedure that we have implemented only dereddens sources with $J-K>1.0$, so that only the highly extinguished sources will be corrected. Furthermore, in order to remove many of the local disc sources and so improve the contrast, all sources with $\left(J-K_{\mathrm{s}}\right)<0.5$ were removed. This means that the inner Galaxy sources will be preferentially corrected, whereas the local disc sources, which are not as reddened, will not be corrected or removed. This approach is roughly consistent with that of Schultheis et al. (1999), except that here each star is individually corrected for extinction before each area is averaged. The star counts corrected for extinction using this method are shown in the lower plot of Fig. 10.

This approach is reasonable because of the very high density of sources in the inner Galaxy. In many regions over $50 \%$ of the sources at a particular magnitude are from the inner Galaxy and so by improving the ratio the vast majority of the detected sources come from a relatively restricted distance range $(8 \pm 3 \mathrm{kpc})$. To have an extinction-corrected apparent $K$ magnitude of +9 , the sources would need to have an absolute magnitude of between -4.5 and -6.2 , which would mean that the typical intrinsic $J-K$ colour is around 1.0. Even including the more luminous sources, the intrinsic $J-K$ will typically be at most 1.3. An error of $\sim 0.2$ magnitudes in the assumed intrinsic colour leads to an error in the extinctioncorrected $K_{\mathrm{s}}$ magnitude of about 0.1 magnitudes, which is negligible. Clearly some disc sources will be included and these will end up with incorrect magnitudes. However, this will be a small proportion of the total sources and their distribution is symmetrical and in principle predictable, so at most there will be a loss of contrast for the inner Galaxy features. Hence for the case where the majority of the sources are relatively concentrated in a certain location along the line of sight this is a straightforward method for recovering the form of the underlying star distribution, at least in the infrared.

It should be noted that this method needs to detect the source in both $J$ and $K_{\mathrm{s}}$, and so can only remove the effect of extinction up to a certain extinction limit. When the extinction becomes too high, the source will

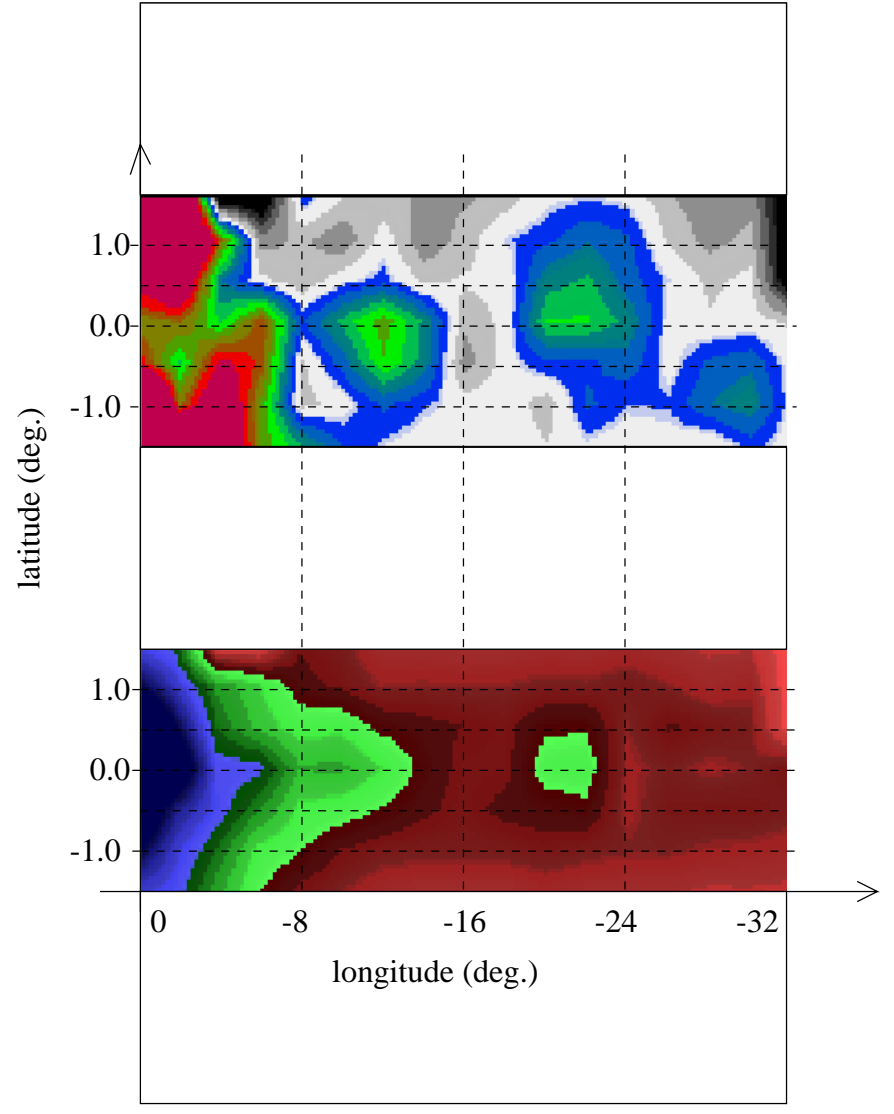

Fig. 10. Map of DENIS star counts binned $\left(\Delta l=2^{\circ}, \Delta b=\right.$ $\left.0.5^{\circ}\right)$ and interpolated. Region $l$ from $0^{\circ}$ to $-32^{\circ} ; b$ from $-1.5^{\circ}$ to $1.5^{\circ}$. Upper: $m_{K_{\mathrm{s}}} \leq 9.0$; lower: $m_{K_{\mathrm{s}} \text {, corrected }} \leq 9.0$ and $(\mathrm{J}-$ $\left.K_{\mathrm{s}}\right)>0.5$.

not be detected at $J$ and so sources will be lost. The $J$ limiting magnitude in DENIS is +16.5 and so the extinction correction limit when making $K$ counts to +9 will be about 20 magnitudes in $V$. The nuclei of the dense dust clouds, particularly near the Galactic Centre, will have far more extinction than this and in these regions hardly any sources will be detected. In general, these regions are small and so when averaging the counts over a reasonable area the losses will be small. However, the extinction-corrected counts shown here should be examined qualitatively, not quantitatively.

The most noticeable effect of the extinction-corrected counts is that a plot that was very patchy is converted into one with a basically smooth distribution of sources. Most of the local troughs have been "filled in" and the peaks disappear. It should also be noted that, unlike the extinction map, the stellar distribution shows no tilt. This may be important for the study of the causes of disc warps and inner tilts in the galaxies, which may be related (Ostriker \& Binney 1989). A force that distorts the gas rather than the stars may indicate an origin related to magnetic fields (Porcel et al. 1997) or accretion of the intergalactic medium (López-Corredoira et al. 2001b). Another possibility is that as the inner disc is truncated there would be 
few stars in the inner disc to be detected, and so even if there were a tilt it would not be seen.

The results of Fig. 10 suggest that the extinction in Fig. 9 is consistent with that derived by the colour-term correction. Hence, by comparing the different maps the following conclusion about the peaks and valleys in Fig. 10 can be made:

$l=0^{\circ}, b=0^{\circ}$ : In the uncorrected counts there was a valley with peaks on either side $\left(b= \pm 1.5^{\circ}\right)$. The maximum source density is expected to be at $l=0^{\circ}, b=0^{\circ}$, but there is too much extinction in this region (over $A_{V} \approx 20$ from Fig. 9). When corrected for extinction, the two peaks are substituted for the one at the centre of the Galaxy;

$l=-5^{\circ}, b=-1^{\circ}$ : In the original counts this local maximum in the star counts coincides with a local minimum in extinction (Fig. 9). When the counts are corrected for extinction, counts from the neighbouring regions increase and the peak disappears. This confirms that this peak is caused by a line of sight which does not pass through a major extinction region;

$l=-8^{\circ}$ to $l=-10^{\circ}, b=0^{\circ}$ : This is a region of high extinction and a dip in the counts. When corrected for extinction the counts recover, the dip disappears and this region becomes part of the spur extending to about $l=-14^{\circ}$

$l=-12^{\circ}$ to $l=-14^{\circ}, b=0^{\circ}$ : The absorption map in Fig. 9 shows that the extinction is lower than in the neighbouring areas and hence that there is a peak in the counts at this location. We suspect that the peak of old $\mathrm{OH} / \mathrm{IR}$ stars at $l=-10^{\circ}$ described by Sevenster (1999) has something to do with the present peak at $l=-12^{\circ}$ for young stars. When the counts are correct for extinction the peak at $l=-12^{\circ}$ is converted into a "spur" in the counts jutting out about 5 degrees from the bulge but only existing very close to the plane. This spur ends at about $l=-14^{\circ}$. It is in the stellar distribution jutting out from the bulge, and fits precisely the far end of the bar described in $\mathrm{H} 00$;

$l=-15^{\circ}$ to $l=-19^{\circ}, b=0^{\circ}$ : This region has significantly lower counts than the neighbouring regions before correcting for extinction, which in this region is apparently high. Calbet et al. (1996) also show this to be a region of high extinction from their analysis of the $C O B E / D I R B E$ surface brightness maps. After correcting for the extinction, the counts do not recover to the same level as the neighbouring regions. This could be explained if this were a zone of extremely high extinction $\left(A_{V}>30 \mathrm{mag}\right)$; however, looking in detail at the distribution of the sources, there is no evidence for the dark "shadows" caused by dense molecular clouds, such as those that are seen towards the Galactic Centre, and so this is unlikely. A more realistic solution is that there are in fact few inner Galaxy sources along these lines of sight. In all lines of sight, sources will be detected from the far side of the disc. These are at a great distance and so are highly reddened.
For most lines of sight towards the inner Galaxy, the inner Galaxy sources are by far the most numerous, so they dominate in any analysis. However, if these source are not present then the far side of the disc will become more important and make the average extinction higher when determined by the methods used here;

$l=-20^{\circ}$ to $l=-24^{\circ}, b=0^{\circ}$ : This maximum extends over a number of degrees in the plane and is still present when the magnitudes are corrected for extinction; hence, this is clearly a real feature in the star distribution and not an artefact caused by patchy extinction in the nearby regions. In H94 this region was proposed to be the far end of the bar. However, this was based on an analysis of the COBE/DIRBE surface brightness maps, which are inherently ambiguous as these offer no information on distance. The data presented in H0O and the new data presented here both show that if there is a bar then the far end is near $l=-12^{\circ}$. The peak, centred at $l=-22^{\circ}$, has more or less the form that is expected for the tangential point to a ring or spiral arm. A full discussion on this is given in the following section;

$l=-30^{\circ}, b=-1^{\circ}$ : This peak almost disappears when the extinction correction is carried out, hence it is just an area of lower extinction.

\section{Discussion}

\subsection{The ring}

The ring by itself cannot explain the form of the counts along the Galactic plane. However this does not mean that there cannot be a ring. More than three quarters of known barred galaxies have rings (Buta 1996), so if the Galaxy is barred then there is a high chance that it will have a ring as well. The prominence of the peak at $l=-22^{\circ}$ indicates that it is caused by an important feature in the inner Galaxy. Its form indicates that it is the tangential point to a ring or spiral arm, and that its location coincides with tangent from the " $3 \mathrm{kpc}$ arm" seen in the CO maps. The $3 \mathrm{kpc}$ arm is an unusual radio feature in the plane with a radial velocity of $-53 \mathrm{~km} \mathrm{~s}^{-1}$ at $l=0^{\circ}$ rather than approximately zero as for the other arms. Furthermore, the tangential point to the Norma arm is at about $l=$ $-30^{\circ}$, yet there is no clear evidence for the arm in the counts. The most likely explanation for the peak at $l=$ $-22^{\circ}$ is that it is the tangential point to a ring, or more probably a pseudo-ring (i.e. the inner arms are very tightly wound around the bar to form what is almost a ring, as in NGC 1433). Sevenster (1999) detected an excess of OH/IR stars at $l=-22^{\circ}$ and suggested that the $3 \mathrm{kpc}$ arm was the cause. He also concluded that this feature is indeed ringlike.

At $l=-22^{\circ}$ the radio tangential point and star-count peaks almost coincide, whereas at tangential points at 
positive longitudes in radio, $l=24^{\circ}$, there is actually a strong dip in the counts. The closest peak to $l=24^{\circ}$ is at $l=27^{\circ}$, hence it is probable that the tangential point to the ring coincides with the end of the bar, which produces the strong peak in the counts seen at the Galactic Centre itself. The reason that the stars and gas are somewhat separated at positive longitudes whilst at negative longitudes they are close together is probably because at positive longitudes the tangential point to the ring almost coincides with the end of the bar. The ring is elliptical and, assuming that the main axis were parallel to the bar, which is not necessarily the case (Sandage \& Bedke 1994), the axial ratio of the ring would be 1:0.76 for the stars and some what higher for the gas. This would compare with the mean axial ratio for rings of $0.81 \pm 0.06$ (Buta 1996). The diameter of the ring would be the same as the bar, hence $8 \mathrm{kpc}$, again very close to the typical values of $9 \mathrm{kpc}$ found in external Galaxies (Freeman 1996).

\subsection{Parameters of the bar}

Combining the results from this paper and those presented in H00 and H94 the principle parameters of the bar can be determined.

The position of the ends of the bar: The near end of the bar is in the first quadrant at $l=27^{\circ}$. At this position there is a sudden change in the stellar density and luminosity functions. The far end of the bar is in the third quadrant and is seen as the spur extending from the bulge to $l=-14^{\circ}$.

Position angle: Using the above positions and assuming that the bar is rectilinear, centred at the Galactic centre and with a width of $500 \mathrm{pc}$, this gives a position angle between the bar and the solar Galactic Centre radius vector of around $40 \pm 5 \mathrm{deg}$. The error comes from the uncertainty in determining the position of the ends but the result is consistent with the other determinations for the in-plane bar (e.g. H00, or Weinberg 1992).

Distance to the bar: The distance to the bar varies from $d=5.7 \mathrm{kpc}$ at the closest position $\left(l=27^{\circ}\right)$ to $d=$ $11.1 \mathrm{kpc}$ at the farthest position $\left(l=-14^{\circ}\right)$.

Length of the bar: The distance from the centre of the Galaxy to the end of the bar is $\sim 0.48 R_{0}$, or $\sim 3.9 \mathrm{kpc}$ (the distance to the Galactic Centre is taken as $R_{0}=$ $7.9 \mathrm{kpc} ; \mathrm{L} 00)$.

Vertical thickness: Figure 4 shows that the counts are very asymmetric at $|b|<0.25^{\circ}$, somewhat less so at $0.5^{\circ}<|b|<1.0^{\circ}$, but symmetric by $1.5^{\circ}<|b|<2.0^{\circ}$. At $|b|<0.25^{\circ}$ there is an average gradient between $l=25^{\circ}$ and $l=-25^{\circ}$ of $\sim 30\left(\mathrm{star} / \mathrm{deg}^{2}\right) / \mathrm{deg}$, which drops to $\sim 20\left(\operatorname{star} / \mathrm{deg}^{2}\right) / \operatorname{deg}$ at $0.5^{\circ}<|b|<1.0^{\circ}$, but is only $\sim 3\left(\right.$ star $\left./ \operatorname{deg}^{2}\right) / \operatorname{deg}$ or less at $1.5^{\circ}<|b|<2.0^{\circ}$. The projected vertical $F W H M$ of the bar is therefore around $1 \mathrm{deg}$ for stars with $M_{K_{\mathrm{s}}}<-6.0$ (or $M_{K_{\mathrm{s}}}<-7.5$ in the farthest positions of the bar).
This implies that the scale height of the young stellar population of the bar discussed here is about $50 \mathrm{pc}$. However, it should be noted that the older component detected in $\mathrm{H} 00$ will probably have a significantly larger scale height.

Star density: From Fig. 4 for $|b|<0.25^{\circ}$, there is an excess of $\sim 1500$ stars $/ \mathrm{deg}^{2}$ over the disc at $l \approx 20^{\circ}$ when compared to $l \approx 30^{\circ}$, and this excess will be mainly due to the bar stars at that location. The extinction at this position is $A_{V} \approx 13 \mathrm{mag}$ (see Fig. 8), and the distance of the bar stars is $d \approx 6 \mathrm{kpc}$. Therefore, assuming a bar width of $\sim 500 \mathrm{pc}$, the density of bar stars up to $m_{K_{\mathrm{s}}}=9.0$ is $\rho \sim 3 \times 10^{-4}$ star $/ \mathrm{pc}^{3}$, i.e. density of bar stars brighter than $M_{K_{\mathrm{s}}}=-6.0$ (mainly late giants, supergiants and a few AGBs). For comparison, the bulge has the same density of stars at about $700 \mathrm{pc}$ from the Galactic Centre in the plane (L00).

\section{Conclusions}

The following features are seen in the plane in the inner regions of the Galaxy $\left(|l|<30^{\circ}\right)$ :

- The star counts are very asymmetric with longitude. There is a major excess of star counts at positive with respect to negative longitudes in the Galactic plane $\left(|b|<1^{\circ}\right)$

- On average, there is more extinction at negative than at positive longitudes in the Galactic plane $\left(|b|<1^{\circ}\right)$;

- Off-plane star counts $\left(1.5^{\circ}<|b|<2.0^{\circ}\right)$ are symmetric in longitude and are nearly constant for $12^{\circ}<|l|<30^{\circ}$;

- There is a peak in the stellar distribution near $l=$ $-22^{\circ}, b=0^{\circ}$, that is not due to a hole in the extinction;

- There is a spur in the counts near the plane extending from the bulge to $l \approx-14^{\circ}$;

- There is a tilt of the gas in the inner disc that is not seen in the stellar component of the central Galaxy.

The asymmetry in the in-plane $2.2 \mu \mathrm{m}$ star counts for $30^{\circ}>l>-30^{\circ}$ cannot be caused by the bulge, and other solutions such as a ring or bar by themselves, spiral arms, etc., simply do not work. Only a ringed barred spiral with an inner truncated disc will naturally reproduce the features seen.

The parameters determined for the bar and ring make the Milky Way a fairly typical barred galaxy. We agree with Sevenster (1999) that NGC 1433 or M95 is a reasonable approximation of what the Galaxy would look like if viewed face on (Figs. 1, 2) giving a revised Hubble type (Sandage \& Tammann 1981) of SBb(s) I-II. The closest part of the bar is in the first quadrant at $l=27^{\circ}$ and the tip at negative Galactic latitudes is near $l=-14^{\circ}$. The bar runs from the bulge to $3.9 \mathrm{kpc}$ and is as dense as the bulge at about $700 \mathrm{pc}$ from the GC, and contains both young and old stars. The inner disc is truncated, with the truncation starting near the radius of the ring. It 
is also proposed that the feature at $l=-22^{\circ}$ is the tangential point to stellar ring. The ring is elliptical and runs through the end of the bar. The spiral arms are probably far weaker than the ring.

Clearly a significant amount of work remains to be done to tie down the parameters with more precision. However, the information now available, particularly from the 2.2 micron star counts, does allow the tentative identification of the Milky Way as an early-type ringed barred Galaxy with a Freeman type II disc.

Acknowledgements. We gratefully acknowledge the anonymous referee for helpful comments. DENIS is the result of a joint effort involving personnel and financial contributions from several Institutes, mostly located in Europe, including the the Instituto de Astrofísica de Canarias (IAC). It has been supported financially mainly by the French Institute National des Sciences de l'Univers, CNRS, and French Education Ministry, the European Southern Observatory, the State of BadenWürttemberg, and the European Commission under a network of the Human Capital and Mobility programme. TMGS observations were made at the Carlos Sánchez Telescope, which is operated by the IAC at its Observatorio del Teide on the island of Tenerife.

\section{References}

Baggett, W. E., Baggett, S. M., \& Anderson, K. S. J. 1996, in IAU Colloq. 157, Barred Galaxies, ed. R. Buta, D. Crocker, \& B. Elmegreen, ASP Conf. Ser., 91, 91

Bertelli, G., Bressan, A., Chiosio, C., Fagotto, F., \& Nasi, E. 1994, A\&AS, 301, 381

Binney, J., Gerhard, O. E., Stark, A. A., Bally, J., \& Uchida, K. I. 1991, MNRAS, 252, 210

Binney, J., Bissantz, N., \& Gerhard, O. 2000, ApJ, 537, L99

Blitz, L., \& Spergel, D. N. 1991a, ApJ, 370, 205

Blitz, L., \& Spergel, D. N. 1991b, ApJ, 379, 631

Borsenberger, J. 1997, in The Impact of Large Scale Near-IR Sky Surveys, ed. F. Garzón, N. Epchtein, A. Omont, B. Burton, \& P. Persi (Kluwer, Dordrecht), 181

Buta, R. 1996, in IAU Colloq. 157, Barred Galaxies, ed. R. Buta, D. Crocker, \& B. Elmegreen, ASP Conf. Ser., 11

Calbet, X., Mahoney, T., Hammersley, P. L., Garzón, F., \& López-Corredoira, M. 1996, ApJ, 457, L27

Cohen, M. 1997, in The Impact of Large Scale Near-IR Sky Surveys, ed. F. Garzón, N. Epchtein, A. Omont, B. Burton, \& P. Persi (Kluwer, Dordrecht), 67

Combes, F. 1996, in IAU Symp. 169, Unsolved Problems of the Milky Way, ed. L. Blitz, \& P. Teuben (Kluwer, Dordrecht), 133

de Vaucouleurs, G. 1964, in IAU Symp. 20, The Galaxy and the Magellanic Clouds, ed. F. J. Kerr, \& A. W. Rodgers (Sydney, Aust. Acad. Sci.), 195

de Vaucouleurs, G. 1970, in IAU Symp. 38, The Spiral Structure of Our Galaxy, ed. W. Becker, \& G. Contopoulos (Reidel, Dordrecht), 18

Dwek, E., Arendt, R. G., Hauser, M. G., et al. 1995, ApJ, 445, 716
Epchtein, N. 1998, in The Impact of Near-Infrared Sky Surveys on Galactic and Extragalactic Astronomy, ed. N. Epchtein (Kluwer, Dordrecht), 3

Epchtein, N., Deul, E., Derriere, S., et al. 1999, A\&A, 349, 236

Freeman, K. C. 1996, in IAU Colloq. 157, Barred Galaxies, ed. R. Buta, D. Crocker, \& B. Elmegreen, ASP Conf. Ser., 91,1

Freudenreich, H. T. 1998, ApJ, 492, 495

Friedli, D. 1996, in IAU Colloq. 157, Barred Galaxies, ed. R. Buta, D. Crocker, \& B. Elmegreen, ASP Conf. Ser., 91, 378

Friedli, D. 1999, in The Evolution of Galaxies on Cosmological Timescales, ASP Conf. Ser. 187, ed. J. E. Beckman, \& T. J. Mahoney (Sheridan Books, San Francisco), 88

Garzón, F. 1999, in The Evolution of Galaxies on Cosmological Timescales, ASP Conf. Ser. 187, ed. J. E. Beckman, \& T. J. Mahoney (Sheridan Books, San Francisco), 31

Garzón, F., Hammersley, P. L., Mahoney, T., et al. 1993, MNRAS, 264, 773

Garzón, F., Hammersley, P. L., Calbet, X., Mahoney, T. J., \& López-Corredoira, M. 1996, in New Extragalactic Perspectives in the New South Africa, ed. D. L. Block, \& J. Mayo Greenberg (Kluwer, Dordrecht), 388

Garzón, F., López-Corredoira, M., Hammersley, P. L., et al. 1997, ApJ, 491, L31

Gerhard, O. E. 1996, in IAU Symp. 169, Unsolved Problems of the Milky Way, ed. L. Blitz, \& P. Teuben (Kluwer, Dordrecht), 79

Glass, I. S. 1999, Handbook of Infrared Astronomy (Cambridge Univ. Press, Cambridge)

Hammersley, P. L., Garzón, F., Mahoney, T., \& Calbet, X. 1994, MNRAS, 269, 753 (H94)

Hammersley, P. L., Garzón, F., Mahoney, T., \& Calbet, X. 1995, MNRAS, 273, 206

Hammersley, P. L., Cohen, M., Garzón, F., Mahoney, T. J., \& López-Corredoira, M. 1999, MNRAS, 308, 333

Hammersley, P. L., Garzón, F., Mahoney, T. J., LópezCorredoira, M., \& Torres, M. A. P. 2000, MNRAS, 317, L45 (H00)

Kent, S. M., Dame, T. M., \& Fazio, G. 1991, ApJ, 378, 131

Kuijken, K. 1996, in IAU Symp. 169, Unsolved problems of the Milky Way, ed. L. Blitz, \& P. Teuben (Kluwer, Dordrecht), 71

Liszt, H. S., \& Burton, W. B. 1980, ApJ, 236, 779

López-Corredoira, M., Garzón F., Hammersley, P. L., Mahoney, T. J., \& Calbet, X. 1997, MNRAS, 292, L15

López-Corredoira, M., Garzón, F., Beckman, J. E., et al. 1999, AJ, 118, 381

López-Corredoira, M., Hammersley, P. L., Garzón, F., Simonneau, E., \& Mahoney, T. J. 2000, MNRAS, 313, 392 (L00)

López-Corredoira, M., Garzón, F., \& Hammersley, P. L. 2001a, MNRAS, 320, 31

López-Corredoira, M., Betancort-Rijo, J., \& Beckman, J. E. 2001b, A\&A, submitted

Nakada, Y., Deguchi, S., Hashimoto, O., et al. 1991, Nature, 353,140

Nakai, N. 1992, PASJ, 44, L27

Ng, Y. K. 1998, in IAU Symp. 184, The Central Regions of the Galaxy and Galaxies, ed. Y. Sofue (Kluwer, Dordrecht), 27 Nikolaev, S., \& Weinberg, M. D. 1997, ApJ, 487, 885

Ohta, K., Hamabe, M., \& Wakamatsu, K. 1990, ApJ, 357, 71

Ostriker, E. C., \& Binney, J. J. 1989, MNRAS, 237, 785

Peters, W. L. 1975, ApJ, 195, 617 
Porcel, C., Battaner, E., \& Jiménez-Vicente, J. 1997, A\&A, Stanek, K. Z. 1995, ApJ, 441, L29 322,103

Ruphy, S., Epchtein, N., Cohen, M., et al. 1997, A\&A, 326, 597

Stanek, K. Z., Mateo, M., Udalski, A., et al. 1994, ApJ, 429, L73

Unavane, M., \& Gilmore, G. 1998, MNRAS, 295, 145

Sandage, A., \& Bedke, J. 1994, The Carnegie Atlas of Galaxies, Carnegie Institution of Washington, Washington DC

Unavane, M., Gilmore, G., Epchtein, N., et al. 1998, MNRAS, 295, 119

Sandage, A., \& Tammann, G. A. 1981, A Revised ShapleyAmes Catalog of Bright Galaxies, Carnegie Institution of Washington, Washington DC

Sanders, D. B., Solomon, P. M., \& Scoville, N. Z. 1984, ApJ, 276,182

Schultheis, M., Ganesh, S., Simon, G., et al. 1999, A\&A, 349, L69

Sevenster, M. N. 1999, MNRAS, 310, 629

Wainscoat, R. J., Cohen, M., Volk, K., Walzer, H. J., \& Schwartz, D. E. 1992, ApJS, 83, 111

Weiland, J. L., Arendt, R. G., Berriman, G. B., et al. 1994, ApJ, 425, L81

Weinberg, M. D. 1992, ApJ, 384, 81

Weiner, B. J., \& Sellwood, J. A. 1999, ApJ, 524, 512

Whitelock, P. A., Feast, M. W., \& Catchpole, R. M. 1991, MNRAS, 248, 276

Sevenster, M. N., Prasenjit, S., Valls-Gabaud, D., \& Fux, R. 1999, MNRAS, 307, 584

Woźniak, P. R., \& Stanek, K. Z. 1996, ApJ, 464, 233

Yuan, C. 1984, ApJ, 281, 600 